\title{
An Improvement of User Authentication Framework for Cloud Computing
}

\author{
Jongho Mun, Jiye Kim, Dongho Won* \\ College of Information and Communication Engineering, Sungkyunkwan University, Korea. \\ * Corresponding author. Tel: +82-31-290-7107; email: dhwon@security.re.kr \\ Manuscript submitted July 15, 2015; accepted December 15, 2015. \\ doi: 10.17706/jcp.11.6.446-454
}

\begin{abstract}
Cloud computing is an emerging computing paradigm in which resources of the computing infrastructure are provided as outsourcing services through the Internet. With cloud computing technique, the users are able to outsource their computing and storage tasks to the cloud servers. In cloud computing environments, the user authentication is very important issue because it provides the authorization while users access their data and cloud services. In 2014, Chen et al. improved Choudhury et al.'s scheme, and claimed that their scheme is more secure and practical remote user authentication scheme for cloud computing. However, we find that Chen et al.'s scheme is still insecure against outsider attack, server impersonation attack, smart card stolen attack and off-line password guessing attack. To overcome this drawback, we propose a robust and more secure user authentication scheme. Finally, we show that our proposed scheme is more secure and supports security properties than Chen et al.'s scheme.
\end{abstract}

Key words: Cloud computing, remote user authentication, smart card.

\section{Introduction}

Cloud Computing [1] is defined that refers to both the applications delivered as services via the Internet and the hardware and system software in the data center that provide those services. Thus, "cloud" can be defined a set of storages, services and interfaces those are provides by servers. Cloud computing allows users store and access all the data and services through the Internet instead of their own computers. The cloud computing can be categorized according to services available on the cloud namely IaaS (Infrastructure as a Service), SaaS (Software as a Service) and PaaS (Platform as a Service) [2]. According to [3], the security issues of cloud system can be classified into four categories: authentication, data integrity, data confidentiality and access control. Above all, the user authentication in cloud computing environments is very important issue because it provides the authorization while users access their data and cloud services. Since Lamport [4] proposed the first password-based authentication scheme over insecure communication in 1981, password-based authentication schemes [5]-[8] have been extensively investigated. However, a problem of password-based authentication scheme is that a server must maintain a password table for verifying the legitimacy of a remote user. Therefore, the server requires additional memory space for storing the password table for verifying user identity. Furthermore, password is generally simple and can be easily broken or forgotten. For this reason, many remote user authentication schemes using smart card [9]-[12] have been proposed. In 2011, Choudhury et al. [13] presented a user authentication frame for cloud computing. Choudhury et al. applies identity authentication with smart card 
for cloud computing. Their scheme verifies user authenticity using two-step verification, which is based on password, smart card and out of band authentication. However, in 2014, Chen et al. [14] found that Choudhury et al's scheme does not provide proper authentication and cannot resist impersonation attack. They proposed an improved scheme of Choudhury et al.'s scheme for cloud computing. However, Chen et al.'s scheme is still insecure. We find that their scheme cannot withstand outsider attack, impersonation attack, smart card stolen attack and off-line password guessing attack as well. Furthermore, Chen et al.'s scheme has no wrong password detection mechanism. To overcome this drawback, we propose a robust and more secure remote user authentication scheme which is an improvement of Chen et al.'s scheme. The remainder of the paper is organized as follows. We begin by reviewing Chen et al.'s remote user authentication scheme in Section 2. In Section 3, we describe security weaknesses of Chen et al.'s scheme. Our proposed scheme is presented in Section 4. Security analysis of our proposed scheme is given in Section 5. Finally, we conclude this paper in Section 6.

\section{Review in Chen et al.'s Scheme}

This section reviews the remote user authentication scheme proposed by Chen et al. in 2014. As previous researches, Chen et al.'s scheme consists of four phases: registration, login, authentication and password change phases which as follows. The notations used in this paper are summarized as Table 1.

Table 1. Notations Used in This Paper

\begin{tabular}{ll}
\hline \hline Notations & Description \\
\hline$A(U)$ & A login user \\
$S$ & The cloud server \\
$S C$ & A smart card \\
$I D, P W$ & Identity and password of the user \\
$N$ & A random number unique to login user \\
$K$ & One-time key \\
$X$ & A user's secret number \\
$Y$ & A server's secret number stored at the server \\
$P$ & A large prime number \\
$G$ & Primitive element in the Galois field $G F(p)$ \\
$h(\cdot)$ & One way hash function \\
$E_{k}(\cdot) / D_{k}(\cdot)$ & The symmetric encryption/decryption function with the key $K$ \\
$\|$ & Concatenation operation \\
$X \rightarrow Y$ & Message $M$ is sent $X$ to $Y$ through public channel \\
$X \Rightarrow Y$ & Message $M$ is sent $X$ to $Y$ through secure channel \\
$\oplus$ & The XOR operation \\
\hline \hline
\end{tabular}

\subsection{Registration Phase}

The registration phase is the initial phase of the scheme. In this phase, user provides appropriate identification details to the cloud server. Then the cloud server issues a smart card to user according user's data.

1) $A$ selects a random number $\mathrm{x}$ and computes $h(P W \oplus x)$.

2) $A \Rightarrow S: I D, h(P W), h(P W \oplus x)$.

3) $S$ checks whether the $I D$ has existed in server. If $I D$ has existed in server, $S$ rejects registration request. Otherwise, $S$ generates $y$ and computes:

$$
\begin{gathered}
I D=h(I D \| y) \\
B=g^{I D+h(P W)+h(y)} \bmod p
\end{gathered}
$$

4) $S \Rightarrow A$ : a smart card. $S$ sends smart card which contains $\{I, B, p, g, h(\cdot)\}$ to user $A$ over secure 
channel.

5) $A$ enters x into his smart card. Now, smart card contains $\{I, B, p, g, h(\cdot), x\}$.

6) $S$ stores $I D$ and $h(P W \oplus x)$ in the server.

\subsection{Login Phase}

This phase is invoked when user wants to login into cloud. To start any conversation, the user must first login to a specific terminal using smart card.

1) $A$ inserts his smart card and inputs $I D$ and $P W$.

2) The smart card computes $C=h\left(I\|h(P W \oplus x)\| T_{u}\right)$ where $T_{u}$ denotes $A$ 's current timestamp.

3) $A \rightarrow S: I D, C, T_{u}$.

\subsection{Authentication Phase}

After receiving the login request messages $\left\{I D, C, T_{u}\right\}$, the server verifies the identity of the user. The procedure is as follows.

1) If $T_{u}{ }^{\prime}-T_{u}>\Delta T, S$ rejects $A$ 's login request. Otherwise, $S$ performs the following computations:

$$
\begin{gathered}
\mathrm{I}^{*}=h(I D \| y) \\
C^{*}=h\left(\mathrm{I}^{*}\|h(P W \oplus x)\| T_{u}\right)
\end{gathered}
$$

where $T_{u}{ }^{\prime}$ is the current timestamp of server and $\Delta T$ is the maximum time interval for transmission delay.

If $C^{*}$ equals $C, S$ accepts the user $A^{\prime}$ s login request and computes, $K^{\prime}=g^{I D+h(y)} \bmod p, h\left(K^{\prime}\right)$ and $R=$

$h\left(K^{\prime} \| T_{S}\right) . T_{S}$ is $S^{\prime}$ s current timestamp. Then, $S$ generates a random number $a$.

2) $S \rightarrow A: E_{h\left(K^{\prime}\right)}\left\{R, T_{S}, a\right\}$.

3) Upon receiving response message, $A$ computes $K^{\prime \prime}=B g^{-h(P W)} \bmod p$ and $h\left(K^{\prime \prime}\right)$. Then, $A$ decrypts $E_{h\left(K^{\prime}\right)}\left\{R, T_{S}, a\right\}$ with $h\left(K^{\prime \prime}\right)$ and gets $\left\{R, T_{s}, a\right\} . A$ checks the timestamp. If $T_{s}$ is invalid, $A$ terminates this session. Otherwise, $A$ computes $R^{\prime}=h\left(K^{\prime \prime} \| T_{S}\right)$ and compares $R^{\prime}$ to the received $R$. If equal, $A$ successfully authenticates $S$ and sends the value $h(a)$ to server $S$.

4) $S$ checks $h(a)$. If $h(a)$ is correct, mutual authentication successes. Now both user $A$ and server $S$ can compute the session key $S_{K}=h\left(K^{\prime} \| a\right)=h\left(K^{\prime \prime} \| a\right)$.

\subsection{Password Change Phase}

The phase is invoked when the user wants to change his/her password.

1) $A$ inserts his/her smart card into card-reader and inputs $I D$ and $P W$.

2) $A \rightarrow S: E_{S_{K}}\left\{h(P W \oplus x)\left\|h\left(P W^{\prime} \oplus x\right)\right\| b\right\} . A$ and $S$ executes the login and authentication phase mentioned above. If $A$ passes the verification, $A$ will send a password change request to $S$ and then submit $h(P W \oplus x)$ and $h\left(P W^{\prime} \oplus x\right)$ where $P W^{\prime}$ is $A^{\prime}$ s new password and $b$ is random number.

3) After receiving password change request, $S$ checks $h(P W \oplus x)$ and replaces it by $h\left(P W^{\prime} \oplus x\right)$.

4) $S \rightarrow A: h(b)$.

5) When receiving response message, A checks $h(b)$. If it is correct, the smart card performs,

$$
\begin{aligned}
& Z=B g^{-I D-h(P W)} \bmod p \\
& B^{\prime}=Z g^{I D+h\left(P W^{\prime}\right)} \bmod p
\end{aligned}
$$

6) $A$ replaces $B$ by $B^{\prime}$ in the smart card. 


\section{Security Analysis of Chen et al.'s Scheme}

In this section, we demonstrate the vulnerability of Chen et al.'s scheme in various communication scenarios.

\subsection{Denial-of-Service via Wrong Password Login}

This is the type of attack when a legal user is denied access to services which are meant for him. Suppose $A$ inserts wrong password $P W$ in login phase. Smart card has no mechanism to detect it, then $A$ sends wrong login request $\left\{I D_{a}, C_{a}{ }^{\prime}, T_{a}\right\}$ as login request, when $S$ checks the equivalence $h\left(I_{a}\left\|h\left(P W_{a} \oplus x\right)\right\| T_{a}\right)=$ ? $C_{a}{ }^{\prime}$. Clearly, it will not hold. As a result, $S$ will terminate the session and $A$ will face the DoS.

\subsection{Outsider Attacks}

Any adversary $O$ who is the legal user and owns a smart card can obtain information $\left\{I_{o}, B_{o}, p, g, h(\cdot\right.$ ), $\left.x_{o}\right\}$ and then he/she can compute $g^{h(y)} \bmod p=B_{o} g^{-h\left(P W_{o}\right)-I D_{o}} \bmod p$. If an adversary $O$ intercepts any user $A^{\prime}$ 's login request message $\left\{I D_{a}, C_{a}, T_{a}\right\}$ and server $S^{\prime}$ s response message $E_{h(K)}\left\{R_{s}, T_{s}, a_{s}\right\}$, then he/she can compute the encryption key $h\left(K^{\prime}\right)$ by calculating $h\left(g^{I D_{a}+h(y)} \bmod p\right)$. Hence, adversary $O$ can decrypt server $S^{\prime}$ s response message and compute the session key $S_{K}=h\left(K^{\prime} \| a\right)$.

\subsection{Server Impersonation Attacks}

If user $A$ sends login request message $\left\{I D_{a}, C_{a}, T_{a}\right\}$ to outsider adversary $O$ which impersonates as the server $S$, then $O$ easily can compute $K^{\prime}=g^{I D_{a}+h(y)} \bmod p$ by using $g^{h(y)} \bmod p$. An adversary $O$ performs following step.

1) The adversary generates a random number $a_{o}$ and computes $h\left(K^{\prime}\right)$ and $R_{o}=h\left(K^{\prime} \| T_{o}\right)$ where $T_{o}$ is $O$ 's current timestamp.

2) $O \rightarrow A: E_{h\left(K^{\prime}\right)}\left\{R_{o}, T_{o}, a_{o}\right\}$. After receiving response message $E_{h\left(K^{\prime}\right)}\left\{R_{o}, T_{o}, a_{o}\right\}$ from $O, A$ will decrypt response message and check the timestamp $T_{o}$ and $R_{o}$. However, $R_{a}{ }^{\prime}$ is equal of $R_{o}$. Therefore, an outsider adversary $O$ can impersonate the server $S$.

\subsection{Smart Card Stolen \& Off-line Password Guessing Attacks}

If an adversary $O$ intercepts legitimate user $A^{\prime}$ s login request message $\left\{I D_{a}, C_{a}, T_{a}\right\}$ and steals $A^{\prime}$ s smart card, he/she can obtain parameters $\left\{I_{a}, B_{a}, p, g, h(\cdot), x_{a}\right\}$. Then, an adversary $O$ can perform off-line password guessing attacks.

1) An adversary guesses any password $P W^{*}$.

2) Then an adversary calculates $h\left(I_{a}\left\|h\left(P W^{*} \oplus x_{a}\right)\right\| T_{a}\right)$ and compares it with $C_{a}$. If the result is equal to $C_{a}$, the adversary infers that $P W^{*}$ is user $A^{\prime}$ s password. Otherwise the adversary selects another password nominee and performs the same processes, until he/she locates the valid password.

\subsection{Requirement of Verifying Table}

The server $S$ must maintain the verifying table that stores each user's $I D$ and $h(P W \oplus x)$ for verifying the legitimacy of the login users in Chen et al.'s proposed scheme. Therefore, the server $S$ requires extra memory space to store the verifying table. Although the one-way hash functions and encryption algorithms are applied to prevent the passwords from being disclosed, the verifying table is still vulnerable. Because any insider adversary who knows the server's secret key $y$ easily can impersonate any user by using $I D$ and $h(P W \oplus x)$. Furthermore, if user wants to change his/her password, then server $S$ should replace $h(P W \oplus x)$ to $h\left(P W^{\prime} \oplus x\right)$ in verifying table.

\section{Our Proposed Scheme}


In this section, we describe more secure remote user authentication. Our improved scheme consists of four phase and works as follows.

\subsection{Registration Phase}

The registration phase is operated when the user $U_{i}$ initially registers to the cloud server $S$ and is described as follows.

1) $U_{i}$ chooses his/her identity $I D_{i}$ and password $P W_{i}$, then computes $h\left(P W_{i}\right)$.

2) $U_{i} \Rightarrow S: I D_{i}, h\left(P W_{i}\right) . U_{i}$ sends $\left\{I D_{i}, h\left(P W_{i}\right)\right\}$ to the server $S$ over a secure channel.

3) Upon receiving registration request message from $U_{i}, S$ generates $N_{i}$ and $y$ where $N_{i}$ is a random number to unique to $U_{i}$. Then, $S$ computes,

$$
\begin{gathered}
F_{i}=h(y) \oplus N_{i} \\
D_{i}=h\left(y \| N_{i}\right) \oplus I D_{i} \\
B_{i}=g^{I D_{i}+h\left(P w_{i}\right)+h\left(N_{i} \| y\right)} \bmod p \\
V_{i}=F_{i} \oplus h\left(I D_{i} \oplus h\left(P W_{i}\right)\right)
\end{gathered}
$$

where $p$ is a large prime number and $y$ is a server $S$ 's secret number stored at the server.

4) $S \Rightarrow A$ : a smart card. $S$ installs $\left\{F_{i}, D_{i}, B_{i}, V_{i}, p, g, h(\cdot)\right\}$ in the smart card and sends the smart card to user $U_{i}$ via secure channel.

\subsection{Login Phase}

If $U_{i}$ wishes to login cloud server $S, U_{i}$ inserts his/her smart card into the card-reader and performs the following steps.

1) $U_{i}$ inputs his/her identity $I D_{i}$ and password $P W_{i}$.

2) Then, smart card computes $F_{i}{ }^{*}=V_{i} \oplus h\left(I D_{i} \oplus h\left(P W_{i}\right)\right)$ and compares $F_{i}{ }^{*}$ with stored $F_{i}$. If it holds, smart card computes,

$$
\begin{gathered}
W_{i}=B_{i} g^{-h\left(P w_{i}\right)} \bmod p \\
C_{i}=h\left(W_{i} \| T_{i}\right)
\end{gathered}
$$

where $T_{i}$ is the current timestamp. Otherwise, smart card rejects login request.

3) $U_{i} \rightarrow S: F_{i}, D_{i}, C_{i}, T_{i}$. Smart card sends login request $\left\{F_{i}, D_{i}, C_{i}, T_{i}\right\}$ to $S$ through a common channel.

\subsection{Authentication Phase}

After receiving the login request message $\left\{F_{i}, D_{i}, C_{i}, T_{i}\right\}$ from $U_{i}$, the server $S$ verifies the identity of the user $U_{i}$. The procedure is as follows.

1) If $T_{u}{ }^{\prime}-T_{u}>\Delta T, S$ rejects $U_{i}{ }^{\prime}$ s login request. Otherwise, $S$ performs the following computations:

$$
\begin{gathered}
N_{i}=F_{i} \oplus h(y) \\
I D_{i}=D_{i} \oplus h\left(y \| N_{i}\right) \\
W_{i}^{*}=g^{I D_{i}+h\left(N_{i} \| y\right)} \bmod p \\
C_{i}^{*}=h\left(W_{i}^{*} \| T_{i}\right)
\end{gathered}
$$


where $T_{i}{ }^{\prime}$ is the current timestamp of server and $\Delta T$ is the maximum time interval for transmission delay.

If $C_{i}{ }^{*}$ equals $C_{i}, S$ accepts the user $U_{i}$ 's login request and computes,

$$
\begin{gathered}
K_{s}=W_{i}{ }^{*} g^{T_{i}} \bmod p \\
h\left(K_{s}\right) \\
R_{S}=h\left(K_{S} \| T_{S}\right)
\end{gathered}
$$

where $T_{S}$ is $S^{\prime}$ s current timestamp. Then, $S$ generates a random number $a$.

2) $S \rightarrow U_{i}: E_{h\left(K_{S}\right.}\left\{R_{S}, T_{S}, a\right\} . S$ encrypts $\left\{R_{S}, T_{S}, a\right\}$ with $h\left(K_{S}\right)$, and sends the response message $E_{h\left(K_{S}\right)}\left\{R_{S}, T_{s}, a\right\}$ to user $U_{i}$.

3) After receiving response message, $U_{i}$ computes,

$$
K_{i}=W_{i} g^{T_{i}} \bmod p
$$

and decrypts $E_{h\left(K_{S}\right)}\left\{R_{S}, T_{S}, a\right\}$ with $h\left(K_{i}\right)$ and gets $\left\{R_{S}, T_{S}, a\right\} . U_{i}$ checks the timestamp. If $T_{S}$ is invalid, $U_{i}$ terminates this session. Otherwise, $U_{i}$ computes $R_{i}=h\left(K_{i} \| T_{s}\right)$ and compares $R_{i}$ to the received $R_{s}$. If equal, $U_{i}$ successfully authenticates $S$.

4) $U_{i} \rightarrow S: h(a) . U_{i}$ computes $h(a)$ and sends $h(a)$ to server $S$.

5) $S$ checks $h(a)$. If $h(a)$ is correct, mutual authentication successes. Now both user $U_{i}$ and server $S$ can compute the session key $S_{K}=h\left(K_{i} \| a\right)=h\left(K_{s} \| a\right)$.

\subsection{Password Change Phase}

If $U_{i}$ wants to change his/her password, he/she inserts his/her own smart card into a card reader, then enters identity $I D_{i}$ and password $P W_{i}$. After receiving identity $I D_{i}$ and password $P W_{i}$, smart card performs the following steps.

1) Smart card computes $F_{i}{ }^{*}=V_{i} \oplus h\left(I D_{i} \oplus h\left(P W_{i}\right)\right)$ and compares $F_{i}{ }^{*}$ with stored $F_{i}$. If it holds, smart card accepts $U_{i}$ to enter a new password $P W_{i}{ }^{*}$. Otherwise, smart card rejects password changing request.

2) After receiving new password $P W_{i}{ }^{*}$, smart card computes,

$$
\begin{gathered}
{B_{i}}^{*}=B_{i} g^{-h\left(P w_{i}\right)+h\left(P W_{i}^{*}\right)} \bmod p \\
V_{i}{ }^{*}=F_{i} \oplus h\left(I D_{i} \oplus h\left(P W_{i}^{*}\right)\right)
\end{gathered}
$$

and updates $B_{i}, V_{i}$ as $B_{i}{ }^{*}, V_{i}{ }^{*}$. Then, $U_{i}$ can use the new password $P W_{i}{ }^{*}$ to login the authentication cloud server $S$.

\section{Security Analysis of Our Proposed Scheme}

In this section, we demonstrate that our scheme can withstand several possible attacks. We also show that our scheme supports several security properties. Our proposed scheme keeps the merits of Chen et al.'s scheme

\subsection{Support User Anonymity}

Suppose an adversary $U_{a}$ intercepts on user $U_{i}$ 's login request message. However, he/she fails to guess the user $U_{i}$ 's identity from $\left\{F_{i}, D_{i}, C_{i}, T_{i}\right\}$. In our proposed scheme, we use random number $N_{i}$ which is the unique to user $U_{i}$ and the timestamp $T_{i}$ in the login phase, then user $U_{i}$ 's login request message is changed each login time. An adversary $U_{a}$ does not know $N_{i}$ and $y$. Thus, our proposed scheme supports 
user anonymity.

\subsection{Resisting Impersonation Attack}

In the our proposed scheme, only $U_{i}$ can compute $W_{i}=B_{i} g^{-h\left(P w_{i}\right)} \bmod p$ and $C_{i}=h\left(W_{i} \| T_{i}\right)$ since only he/she has the secrets $N_{i}$ and password $P W_{i}$ and $S$ can compute $N_{i}=F_{i} \oplus h(y)$ and $I D_{i}=$ $D_{i} \oplus h\left(y \| N_{i}\right)$ since only he/she has the secrets $y$. The authentication server $S$ authenticates $U_{i}$ by checking $h\left(g^{I D_{i}+h\left(N_{i} \| y\right)} \bmod p \| T_{i}\right)=$ ? $C_{i}$ and the remote user $U_{i}$ authenticates $S$ by checking $\mathrm{h}\left(K_{i} \| T_{s}\right)=$ ? $R_{s}$. Thus, our proposed scheme can resist impersonation attacks.

\subsection{Resisting Smart Card Stolen Attack}

If an adversary $U_{a}$ steals $U_{i}$ 's smart card, then $U_{a}$ can extract security parameters $\left\{F_{i}, D_{i}, B_{i}, V_{i}, p, g, h(\cdot)\right\}$ from legitimate user $U_{i}$ 's smart card. However, this information does not help them. He/she cannot obtain any information of $U_{i}$ 's $I D_{i}$ and $P W_{i}$ because these values are protected by secret parameters. An identity $I D_{i}$ in $D_{i}\left(=h\left(y \| N_{i}\right) \oplus I D_{i}\right)$ is protected by $S^{\prime}$ s long-term secret key $y$ and the collision resistance one-way hash function $h(\cdot)$, and a password $P W_{i}$ in $V_{i}\left(=F_{i} \oplus h\left(I D_{i} \oplus P W_{i}\right)\right)$, $B_{i}\left(=g^{I D_{i}+h\left(P w_{i}\right)+h\left(N_{i} \| y\right)} \bmod p\right)$ is encrypted with $I D_{i}$. Therefore, the our proposed scheme can resist smart card stolen attack.

\subsection{Resisting Replay Attacks}

In the proposed authentication scheme, an adversary cannot correctly modifies $\left\{F_{i}, D_{i}, C_{i}, T_{i}\right\}$ and $E_{h\left(K_{s}\right)}\left\{R_{s}, T_{s}, a\right\}$ without $I D_{i}, P W_{i}, N_{i}$ and $y$, where $I D_{i}=D_{i} \oplus h\left(y \| N_{i}\right), N_{i}=F_{i} \oplus h(y)$ and $C_{i}=h\left(W_{i} \| T_{i}\right)$. When and an adversary $U_{a}$ tries to use the previous message $\left\{F_{i}, D_{i}, C_{i}, T_{i}\right\}$ to login $S$ or $E_{h\left(K_{S}\right)}\left\{R_{S}, T_{S}, a\right\}$ to response $U_{i}$, a failed adversary will be detected by checking the invalid timestamp $T_{i}$ and $T_{s}$. Thus, the proposed authentication scheme is secure against the replay attack.

\subsection{Comparison of Security Properties}

We compare the proposed scheme with the scheme of Choudhury et al. [13], Chen et al. [14] regarding resistance to possible attacks as depicted by Table 2 . Our proposed scheme resists all those attacks to which the previous schemes are susceptible.

Table 2. Comparison of Security Properties

\begin{tabular}{lccc}
\hline \hline Security Properties & Choudhury et al. & Chen et al. & Our scheme \\
\hline User impersonation attacks & No & No & Yes \\
Server impersonation attacks & No & No & Yes \\
Off-line password guessing Attacks & Yes & No & Yes \\
Denial-of-service attacks & Yes & No & Yes \\
Smart card stolen attacks & No & No & Yes \\
Modification attacks & No & No & Yes \\
Replay attacks & Yes & Yes & Yes \\
Support mutual authentication & Yes & Yes & Yes \\
Support user anonymity & Yes & No & Yes \\
Wrong password detection by SC & Yes & No & Yes \\
\hline \hline
\end{tabular}

\section{Conclusion}

In 2014, Chen et al. proposed an enhanced scheme of Choudhury et al.'s scheme and demonstrated it is resistance to famous attacks such as impersonation attacks and out of band attacks. However, Chen et al.'s scheme is still insecure. Furthermore, their scheme has no wrong password detection mechanism then may deduce the DoS problem. In this paper, we shown how their scheme can suffer from outsider attacks, server 
impersonation attacks and smart card stolen attacks and proposed an improved protocol for authentication scheme that keeps the similar properties of Chen et al's scheme and make it more secure. The security analysis explains that our improved scheme rectifies the vulnerabilities of Chen et al.'s scheme.

\section{Acknowledgment}

This work was supported by Institute for Information \& communications Technology Promotion(IITP) grant funded by the Korea government(MSIP) (No.R0126-15-1111, The Development of Risk-based Authentication· Access Control Platform and Compliance Technique for Cloud Security)

\section{References}

[1] Armbrust, M., Fox, A., Griffith, R., Joseph, A., Katz, R., Konwinski, A., Lee, G., Patterson, D., Rabkin, A., Stoica, I., \& Zaharia, M. (2010). A view of cloud computing. Communication of the ACM, 53, 50-58.

[2] Smith, E., et al. (2010). The management of security in cloud computing. Proceedings of Information Security for South Africa (pp. 1-7).

[3] Huang, C., Qin, Z., \& Kuo, C. (2011). Multimedia storage security in cloud computing: An overview. Proceedings of IEEE 13th International Workshop on Multimedia Signal Processing (pp. 1-6).

[4] Lamport, L. (1981). Password authentication with insecure communication. Communication of the ACM, 24, 770-772.

[5] Gennaro, R., \& Lindell, Y., (2006). A framework for password-based authenticated key exchange. ACM Transactions on Information and System Security, 9, 181-234.

[6] Yang, Y., Deng, R., \& Bao, F. (2006). A practical password-based two-server authentication and key exchange system. IEEE Transactions on Dependable and Secure Computing, 3(2), 105-114.

[7] Jeun, I., Kim, M., \& Won, D. (2012). Enhanced password-based user authentication using smart phone. Advances in Grid and Pervasive Computing, 7296, 350-360.

[8] Lee, Y., \& Won, D. (2013). On the use of a hash function in a 3-party password-based authenticated key exchange protocol. Grid and Pervasive Computing, 7681, 730-736.

[9] Lee, J., Ryu, S., \& Yoo, K. (2002). Fingerprint-based remote user authentication scheme using smart cards. Electronics Letters, 38, 554-555.

[10] Yi, W., Kim, S., \& Won, D. (2009). Smart card based AKE protocol using biometric information in pervasive computing environments. Computational Science and Its Applications, 5593, 182-190.

[11] Lee, Y. (2012). A new dynamic ID-based user authentication scheme to resist smart-card-theft-attack. Applied Mathematics and Information Sciences, 6, 355-361.

[12] Mun, J., Jin, Q., Jeon, W., \& Won, D. (2013). An improvement of secure remote user authentication scheme using smart cards. Proceedings of International Conference on IT Convergence and Security (pp. 1-4).

[13] Choudhury, A., Kumar, P., Sain, M., Lim, H., \& Lee, H. (2011). A strong user authentication framework for cloud computing. Proceedings of 2011 IEEE Asia-Pacific Services Computing Conference (pp. 110-115).

[14] Chen, N., \& Jiang, R. (2014). Security analysis and improvement of user authentication framework for cloud computing. Journal of Networks, 9, 198-203.

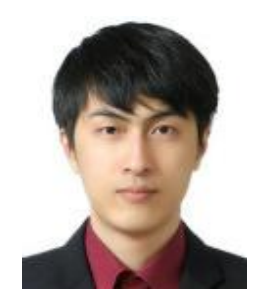

Jongho Mun was born in Jinju, Korea on November 16, 1984. He received the B.S. degree in electrical and computer engineering from Sungkyunkwan University, Korea, in 2012 and the M.S. degree in electrical and computer engineering from Sungkyunkwan University, Korea, in 2014. He also worked as a malware analyzer in SECUI between 2014 and 2015. 
$\mathrm{He}$ is currently pursuing the Ph.D. degree in electrical and computer engineering at Sungkyunkwan University. His current research interest includes cryptography, malware, forensic, and authentication or key management protocols.

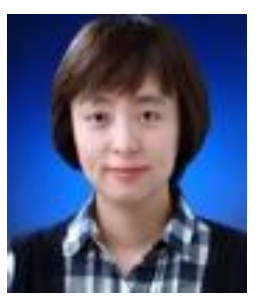

Jiye Kim was born in Seoul, Korea on December 2, 1976. She received the B.S. degree in information engineering from Sungkyunkwan University, Korea, in 1999 and the M.S. degree in computer science education from Ehwa University, Korea, in 2007. She also worked as a software engineer for mobile phone manufacturers in Korea or Japan between 1999 and 2013. She is currently pursuing the Ph.D. degree in electrical and computer engineering at Sungkyunkwan University. Her current research interest includes cryptography, authentication or key management protocols, wireless sensor networks, and mobile security.

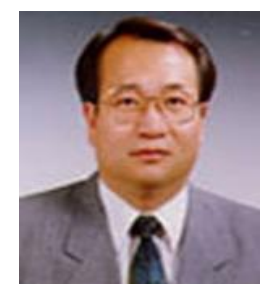

Dongho Won was born in Seoul, Korea on September 23, 1949. He received BSC, MSC and Ph.D. in electronic engineering from Sungkyunkwan University, Korea. After working in Electronics and Telecommunication Research Institute for two years, he joined Sungkyunkwan University, where he is currently a leader professor at Information and Communication Engineering. He also served as the president of Korea Institute of Information Security and Cryptography. He was the Program Committee Chair Man of 8th International Conference on Information Security and Cryptography in 2005. His research interests are cryptology and information security. 